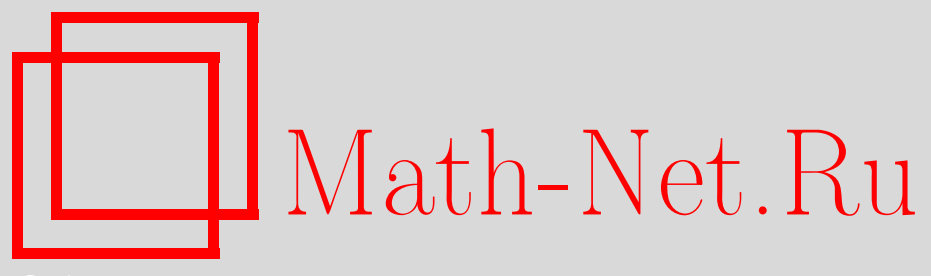

В. И. Лотов, А. С. Тарасенко, Об асимптотике среднего времени пребывания случайного блуждания на полуоси, Изв. РАН. Сер. матем., 2015, том 79, выпуск 3, 23-40

DOI: https://doi.org/10.4213/im8176

Использование Общероссийского математического портала Math-Net.Ru подразумевает, что вы прочитали и согласны с пользовательским соглашением http://www . mathnet.ru/rus/agreement

Параметры загрузки:

IP : 3.81 .55 .215

26 апреля 2023 г., 15:05:13

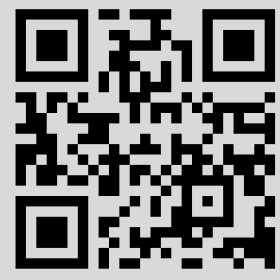


УДК 519.21

\author{
В. И. Лотов, А. С. Тарасенко
}

\title{
Об асимптотике среднего времени пребывания случайного блуждания на полуоси
}

\begin{abstract}
Найдены асимптотические разложения математического ожидания времени пребывания траектории случайного блуждания с нулевым сносом выше растущего уровня. На распределение скачков блуждания налагается условие Крамера о существовании экспоненциального момента.

Библиография: 13 наименований.
\end{abstract}

Ключевые слова: случайное блуждание, время пребывания, асимптотический анализ.

DOI: $10.4213 / \mathrm{im} 8176$

\section{§ 1. Введение. Формулировка основных результатов}

Пусть $X_{1}, X_{2}, \ldots$ - последовательность независимых одинаково распределенных случайных величин, и пусть $S_{n}=X_{1}+\cdots+X_{n}, n \geqslant 1$. Для произвольного $b \geqslant 0$ введем время пребывания случайного блуждания $\left\{S_{k}, k=1, \ldots, n\right\}$ на полуоси $(b, \infty)$ следующим образом:

$$
T_{n}(b)=\sum_{k=1}^{n} I_{\left\{S_{k}>b\right\}},
$$

где $I_{A}-$ индикатор события $A$.

Целью настоящей работы является изучение асимптотического поведения математического ожидания $\mathrm{E} T_{n}\left(b_{n}\right)$ при $n \rightarrow \infty$, где $b_{n} \rightarrow \infty$ с определенной скоростью, соответствующей области больших уклонений.

Изучение распределения времени пребывания случайного блуждания на отрезке или на полуоси является весьма трудной задачей, и ему посвящено много работ. Для простейших блужданий ряд результатов о времени пребывания может быть получен с помощью комбинаторных методов. Хорошо известен классический закон арксинуса, характеризующий предельное поведение распределения $T_{n}(0)$ (см., например, [1], [2]). Известны также предельные теоремы о времени пребывания, основанные на использовании сходимости распределений функционалов от траекторий случайного блуждания к распределению соответствующих функционалов от предельных процессов. Весьма подробная библиография и результаты этого направления исследований содержатся в работах [3], [4]. Асимптотические разложения для $\mathrm{P}\left(T_{n}\left(b_{n}\right)=k\right)$ при $n \rightarrow \infty$, $b_{n}=o(n)$ найдены в [5].

Работа выполнена при финансовой поддержке РФФИ (грант № 13-01-00046). 
Отметим, что для нахождения среднего времени, проведенного траекторией в отрезке $[a, b]$, достаточно вычесть из $n$ суммарное среднее время, проведенное на полуосях выше уровня $b$ и ниже уровня $a$.

Везде в настоящей работе предполагается выполненным следующее условие:

(C) $\mathrm{E} X_{1}=0$ и $\left|\mathrm{E} e^{\lambda X_{1}}\right|<\infty$ при $|\operatorname{Re} \lambda| \leqslant \beta, \beta>0$.

Ясно, что

$$
\mathrm{E} T_{n}\left(b_{n}\right)=\sum_{k=1}^{n} \mathrm{P}\left\{S_{k}>b_{n}\right\},
$$

поэтому асмиптотическое поведение $\mathrm{E} T_{n}\left(b_{n}\right)$ можно исследовать, пользуясь известными результатами о вероятностях больших уклонений для сумм независимых слагаемых. Пусть $\psi(\lambda)=\mathrm{E} e^{\lambda X_{1}}, \mathrm{D} X_{1}=\sigma^{2}$ и

$$
\Lambda(\alpha)=\sup _{\lambda \in \mathbb{R}}(\alpha \lambda-\ln \psi(\lambda))
$$

- известная функция уклонений. Всюду далее $\alpha_{n} \underset{n \rightarrow \infty}{\sim} \beta_{n}$ будет означать, что $\alpha_{n}=\beta_{n}(1+o(1))$.

Теорема 1. Пусть выполнено условие (C), и пусть $b_{n}$ - монотонная последовательность такая, что $\frac{b_{n}}{\sqrt{n}} \rightarrow \infty u \frac{b_{n}}{n} \rightarrow 0$ при $n \rightarrow \infty$. Тогда

$$
\mathrm{E} T_{n}\left(b_{n}\right) \underset{n \rightarrow \infty}{\sim} \sqrt{\frac{2}{\pi}} \frac{n^{\frac{5}{2}} \sigma^{3}}{b_{n}^{3}} e^{-n \Lambda\left(\frac{b_{n}}{n}\right)} .
$$

Приведенное ниже доказательство этой теоремы основано на известных оценках вероятностей больших уклонений.

Далее рассмотрим вопрос о получении полных асимптотических разложений для $\mathrm{E} T_{n}\left(b_{n}\right)$. Не исключено, что их также можно получить с помощью теорем об асимптотических разложениях вероятностей больших уклонений, хотя этот подход представляется весьма трудным. Для нахождения полных асимптотических разложений мы избрали другой путь, о котором пойдет речь ниже и для реализации которого ряд предварительных результатов уже получен ранее.

Отметим, что изучение времени пребывания, несомненно, относится к граничным задачам для случайных блужданий. Как и в других работах, посвященных исследованиям в граничных задачах, асимптотический анализ $\mathrm{E} T_{n}\left(b_{n}\right)$ в условиях Краме́ра может быть проведен с помощью так называемого факторизационного метода. Этот метод технически весьма сложен, однако именно он позволяет получить в ряде граничных задач полные асимптотические разложения изучаемых величин. Этим и будем заниматься в оставшейся части работы.

Напомним, что факторизационный метод разбивается на несколько этапов. Исследование начинается с нахождения тождеств, связывающих двойные или даже тройные преобразования Лапласа-Стилтьеса над распределением исследуемого граничного функционала с компонентами известной факторизации 
Винера-Хопфа, или, по другой терминологии, с двойными преобразованиями над распределениями лестничных величин. Как правило, в общем случае эти тождества не приводят к выражениям, пригодным для дальнейшего их обращения. В связи с этим на втором этапе проводится асимптотический анализ полученных выражений при условии, что граница рассматриваемого множества удаляется. На этом пути выделяются главные части полученных на первом этапе преобразований и оценивается погрешность такого приближения; она оказывается пренебрежимо малой при выполнении условия Крамера для распределения скачков блуждания. Этот этап является достаточно сложным. Здесь требуется в полной мере использовать весьма тонкие свойства компонент факторизации. Найденные главные части преобразований имеют уже сравнительно простую структуру, доступную для последующего асимптотического обращения, что и делается на третьем этапе. Обращение может осуществляться с помощью контурного интегрирования с применением метода перевала (см. [6], [7]) или прямыми вычислениями (см. [8]).

Применительно к исследованию времени пребывания первый этап выполнен в [9], где найдены факторизационные тождества для тройных преобразований над совместным распределением пары $\left(T_{n}, S_{n}\right)$. Затем в [10] произведен асимптотический анализ полученных в [9] факторизационных представлений при условии, что $b \rightarrow \infty$, с целью выделения более просто устроенных главных частей. Тем самым реализован второй этап исследований.

Мы продемонстрируем одно из возможных продолжений [9] и [10], что соответствует третьему этапу. Одним из результатов [10] явилось нахождение асимптотического представления (см. формулу (14) ниже) для производящей функции

$$
r(s)=\sum_{n=1}^{\infty} s^{n} \mathrm{E} T_{n}(b), \quad|s|<1,
$$

которое действует в некоторой окрестности единицы и представляет собой довольно просто устроенную главную часть и остаточный член, который является экспоненциально малым по сравнению с главной частью, если $b \rightarrow \infty$. Дальнейшая схема действий такова. Для нахождения коэффициентов степенного ряда $r(s)$ применяется известный метод контурного интегрирования, при этом выясняется, что при $b \rightarrow \infty$ основной вклад будет вносить интеграл только по той части контура, которая лежит в окрестности единицы. Таким образом, все сводится к работе с главной частью асимптотического представления функции $r(s)$, которое действует как раз в окрестности единицы. Асимптотическое исследование полученного таким образом интеграла проводится методом перевала, осложненного наличием полюса третьего порядка вблизи точки перевала. Это обстоятельство составляет основную техническую трудность работы.

Сформулируем полученный на этом пути результат.

ТЕОРема 2. Пусть распределение $X_{1}$ содержит абсолютно непрерывную компоненту, выполнено условие $(\mathrm{C})$, и пусть $\left\{b_{n}\right\}_{n \geqslant 0}$ - последовательность положительных чисел такая, что $b_{n}=o(n) u \frac{b_{n}}{n^{1 / 2+\varepsilon}} \rightarrow \infty$ рри $n \rightarrow \infty$ для 
некоторого $\varepsilon>0$. Тогда для любого иелого $N \geqslant 1$ при $n \rightarrow \infty$ имеет место разложение

$$
\mathrm{E} T_{n}\left(b_{n}\right)=\frac{e^{n d\left(\tau_{n}\right)}}{2 \pi \sqrt{n} t_{n}^{3}}\left(\sum_{k=0}^{2 N-2} \frac{\left(\nu_{n} n\right)^{-\frac{k}{2}}}{t_{n}^{k}} P_{k}(n)+O\left(\frac{n^{-N}}{t_{n}^{2 N}}\right)\right)
$$

Здесъ $\tau_{n}=\frac{b_{n}}{n}, t_{n}=t\left(\tau_{n}\right), \nu_{n}=\nu\left(\tau_{n}\right), d(\tau), t(\tau)$ u $\nu(\tau)$ - определяемые ниже функиии, аналитические в окрестности нуля,

$$
\begin{gathered}
d(\tau)=-\frac{\tau^{2} \psi_{1}^{2}}{4}+\frac{3 \tau^{3} \psi_{1}^{3} \psi_{2}}{4}+O\left(\tau^{4}\right), \quad t(\tau)=-\frac{\tau \psi_{1}}{2}\left(1-\tau \psi_{2}\right)+O\left(\tau^{3}\right), \\
\nu(\tau)=1+\psi_{2} \tau+O\left(\tau^{2}\right), \\
\psi_{1}=\sqrt{\frac{2}{\sigma^{2}}}, \quad \psi_{2}=\frac{\mu_{3}}{3 \sigma^{4}}, \quad \mu_{k}=\mathrm{E} X_{1}^{k}, \\
P_{k}(n)=\sum_{\substack{l+m \leqslant 2 N-k-2 \\
l+m \equiv k(\bmod 2) \\
l \geqslant 0, m \geqslant 0}} c_{l m k} \Gamma\left(\frac{l+3 m+k+1}{2}\right) \nu_{n}^{-\frac{l+3 m}{2}} n^{-\frac{l+m}{2}},
\end{gathered}
$$

$c_{l m k}$ - коэффициенты разложения в ряд Тейлора некоторой известной функиии, определяемые далее в (19).

В качестве следствия теоремы 2 приведем аппроксимацию $\mathrm{E} T_{n}\left(b_{n}\right)$ главным членом полученного разложения.

СлеДСтвиЕ 1. Пусть выполнены условия теоремы 2. Тогда при $n \rightarrow \infty$

$$
\mathrm{E} T_{n}\left(b_{n}\right)=\sqrt{\frac{2}{\pi}} \frac{n^{\frac{5}{2}} \sigma^{3}}{b_{n}^{3}} e^{n d\left(\tau_{n}\right)}(1+o(1))
$$

Ясно, что это соотношение повторяет результат теоремы 1 , однако в данном случае он следует из теоремы 2 , доказанной при более ограничительных условиях.

\section{§ 2. Доказательство теоремы 1}

Воспользуемся представлением (1). Известно (см., например, [12, гл. 6, следствие 1.1]), что в наших условиях

$$
\mathrm{P}\left(S_{k}>x\right) \underset{k \rightarrow \infty}{\sim} \frac{\sigma \sqrt{k}}{x \sqrt{2 \pi}} e^{-k \Lambda\left(\frac{x}{k}\right)}
$$

равномерно по всем $\frac{x}{n} \in\left[0, \alpha_{+}\right)$, где

$$
\alpha_{+}:=\frac{\psi^{\prime}\left(\lambda_{+}-0\right)}{\psi\left(\lambda_{+}-0\right)}, \quad \lambda_{+}:=\sup \left\{\lambda>0: \mathrm{E} e^{\lambda X_{1}}<\infty\right\} .
$$

В силу того, что $\frac{\sqrt{n}}{b_{n}} \underset{n \rightarrow \infty}{\longrightarrow} 0$ и $\frac{b_{n}}{n} \underset{n \rightarrow \infty}{\longrightarrow} 0$, можно выбрать последовательность положительных чисел $\varepsilon_{n}$, достаточно медленно стремящуюся к нулю, такую, что $\frac{n}{\varepsilon_{n} b_{n}^{2}} \underset{n \rightarrow \infty}{\longrightarrow} 0$ и $\frac{b_{n}}{\varepsilon_{n} n} \underset{n \rightarrow \infty}{\longrightarrow} 0$. 
Найдем сначала асимптотику суммы $\sum_{k=K_{n}}^{n} \mathrm{P}\left\{S_{k}>b_{n}\right\}$, где $K_{n}:=\left[n-\frac{n^{2}}{\varepsilon_{n} b_{n}^{2}}\right]$ (здесь берется целая часть числа).

Известно (см. [11, с. 231]), что в наших условиях справедливо разложение функции $\Lambda(\alpha)$ в ряд в некоторой окрестности нуля: $\Lambda(\alpha)=\frac{\alpha^{2}}{2 \sigma^{2}}+\sum_{i=3}^{\infty} a_{i} \alpha^{i}$, где $a_{i}=\frac{\Lambda^{(i)}(0)}{i !}$. Положим

$$
\lambda(\alpha):=\sum_{i=3}^{\infty} \frac{\Lambda^{(i)}(0)}{i !} \alpha^{i}=\Lambda(\alpha)-\frac{\alpha^{2}}{2 \sigma^{2}} .
$$

Покажем, что равномерно по всем $k \in\left[K_{n}, n\right]$ выполняется

$$
\exp \left\{k \lambda\left(\frac{b_{n}}{k}\right)\right\} \underset{n \rightarrow \infty}{\sim} \exp \left\{n \lambda\left(\frac{b_{n}}{n}\right)\right\}
$$

или, что то же самое,

$$
k \sum_{i=3}^{\infty} a_{i}\left(\frac{b_{n}}{k}\right)^{i}-n \sum_{i=3}^{\infty} a_{i}\left(\frac{b_{n}}{n}\right)^{i}=n \sum_{i=3}^{\infty} a_{i}\left(\frac{b_{n}^{i}}{k^{i}}-\frac{b_{n}^{i}}{n^{i}}\right)-(n-k) \sum_{i=3}^{\infty} a_{i}\left(\frac{b_{n}}{k}\right)^{i} \rightarrow 0 .
$$

Оценим последнюю сумму в этом выражении:

$$
\left|(n-k) \sum_{i=3}^{\infty} a_{i}\left(\frac{b_{n}}{k}\right)^{i}\right| \leqslant \frac{C n^{2}}{\varepsilon_{n} b_{n}^{2}} \frac{b_{n}^{3}}{\left(n-\frac{n^{2}}{\varepsilon_{n} b_{n}^{2}}\right)^{3}}=\frac{C b_{n}}{\varepsilon_{n} n} q_{n}^{3} \rightarrow 0,
$$

где $q_{n}:=1-\frac{n}{\varepsilon_{n} b_{n}^{2}} \underset{n \rightarrow \infty}{\longrightarrow} 1$. Через $C, C_{1}, C_{2}, \ldots$ всюду обозначаются положительные константы, возможно, различные для разных выкладок. Далее, из формулы конечных приращений для функции $f(t)=t^{i}$ следует

$$
y^{i}-x^{i}=f(y)-f(x) \leqslant f^{\prime}(y)(y-x)=i y^{i-1}(y-x), \quad y>x .
$$

Поэтому для $k \in\left[K_{n}, n\right]$ имеем

$$
\frac{1}{k^{i}}-\frac{1}{n^{i}}=\frac{n^{i}-k^{i}}{n^{i} k^{i}} \leqslant i n^{i-1} \frac{n^{2}}{\varepsilon_{n} b_{n}^{2}} \frac{1}{n^{2 i}\left(1-\frac{n}{\varepsilon_{n} b_{n}^{2}}\right)^{i}}=\frac{i}{q_{n}^{i}} \frac{n}{\varepsilon_{n} b_{n}^{2}} \frac{1}{n^{i}} .
$$

Следовательно, для $n$ таких, что $q_{n} \geqslant \frac{1}{2}$, имеем

$$
\frac{b_{n}^{i}}{k^{i}}-\frac{b_{n}^{i}}{n^{i}} \leqslant \frac{n}{\varepsilon_{n} b_{n}^{2}} \frac{i}{q_{n}^{i}} \frac{b_{n}^{i}}{n^{i}} \leqslant \frac{n}{\varepsilon_{n} b_{n}^{2}} i \frac{\left(2 b_{n}\right)^{i}}{n^{i}} .
$$

Поскольку ряд $\sum_{i=3}^{\infty}\left|a_{i}\right| i(2 \alpha)^{i}$ сходится в некоторой окрестности нуля наряду с $\sum_{i=3}^{\infty}\left|a_{i}\right| \alpha^{i}$, из соотношения (4) следует

$$
n\left|\sum_{i=3}^{\infty} a_{i}\left(\frac{b_{n}^{i}}{k^{i}}-\frac{b_{n}^{i}}{n^{i}}\right)\right| \leqslant \frac{n^{2}}{\varepsilon_{n} b_{n}^{2}} \sum_{i=3}^{\infty}\left|a_{i}\right| i \frac{\left(2 b_{n}\right)^{i}}{n^{i}} \leqslant C \frac{n^{2}}{\varepsilon_{n} b_{n}^{2}} \frac{b_{n}^{3}}{n^{3}}=C \frac{b_{n}}{\varepsilon_{n} n} \rightarrow 0 .
$$

Таким образом, соотношение (3) установлено, и мы имеем

$$
\sum_{k=K_{n}}^{n} \mathrm{P}\left\{S_{k}>b_{n}\right\} \underset{n \rightarrow \infty}{\sim} \frac{\sigma e^{-n \lambda\left(\frac{b_{n}}{n}\right)}}{b_{n} \sqrt{2 \pi}} \sum_{k=K_{n}}^{n} \sqrt{k} e^{-\frac{b_{n}^{2}}{2 k \sigma^{2}}} .
$$


Нетрудно видеть, что

$$
\sum_{i=K_{n}}^{n} \sqrt{i} e^{-\frac{b_{n}^{2}}{2 i \sigma^{2}}} \underset{n \rightarrow \infty}{\sim} \int_{K_{n}}^{n} \sqrt{t} e^{-\frac{b_{n}^{2}}{2 t \sigma^{2}}} d t
$$

Покажем, что

$$
\int_{K_{n}}^{n} \sqrt{t} e^{-\frac{b_{n}^{2}}{2 t \sigma^{2}}} d t \underset{n \rightarrow \infty}{\sim} \frac{2 \sigma^{2} n^{\frac{5}{2}}}{b_{n}^{2}} e^{-\frac{b_{n}^{2}}{2 \sigma^{2} n}}
$$

откуда будет следовать

$$
\sum_{k=K_{n}}^{n} \mathrm{P}\left\{S_{k}>b_{n}\right\} \underset{n \rightarrow \infty}{\sim} \frac{\sigma e^{-n \lambda\left(\frac{b_{n}}{n}\right)}}{b_{n} \sqrt{2 \pi}} \frac{2 \sigma^{2} n^{\frac{5}{2}}}{b_{n}^{2}} e^{-\frac{b_{n}^{2}}{2 \sigma^{2} n}}=\sqrt{\frac{2}{\pi}} \frac{n^{\frac{5}{2}} \sigma^{3}}{b_{n}^{3}} e^{-n \Lambda\left(\frac{b_{n}}{n}\right)} .
$$

После замены $s=\frac{1}{t}$ приходим к интегралу

$$
\begin{aligned}
\int_{1 / n}^{1 / K_{n}} \frac{1}{s^{\frac{5}{2}}} e^{-\frac{b_{n}^{2}}{2 \sigma^{2}} s} d s & =-\int_{1 / n}^{1 / K_{n}} \frac{2 \sigma^{2}}{b_{n}^{2} s^{\frac{5}{2}}} d\left(e^{-\frac{b_{n}^{2}}{2 \sigma^{2}} s}\right) \\
= & -\left.\frac{2 \sigma^{2}}{b_{n}^{2} s^{\frac{5}{2}}} e^{-\frac{b_{n}^{2}}{2 \sigma^{2}} s}\right|_{1 / n} ^{1 / K_{n}}-\frac{5}{2} \int_{1 / n}^{1 / K_{n}} \frac{2 \sigma^{2}}{b_{n}^{2} s^{\frac{7}{2}}} e^{-\frac{b_{n}^{2}}{2 \sigma^{2}} s} d s \\
= & \frac{2 \sigma^{2} n^{\frac{5}{2}}}{b_{n}^{2}} e^{-\frac{b_{n}^{2}}{2 \sigma^{2} n}}-\left(\frac{2 \sigma^{2} K_{n}^{\frac{5}{2}}}{b_{n}^{2}} e^{-\frac{b_{n}^{2}}{2 \sigma^{2} K_{n}}}+\frac{5}{2} \int_{1 / n}^{1 / K_{n}} \frac{2 \sigma^{2}}{b_{n}^{2} s^{\frac{7}{2}}} e^{-\frac{b_{n}^{2}}{2 \sigma^{2}} s} d s\right) .
\end{aligned}
$$

Напомним, что $K_{n} \underset{n \rightarrow \infty}{\sim} n$, поэтому

$$
\begin{aligned}
& \exp \left\{\frac{-b_{n}^{2}}{2 \sigma^{2} K_{n}}\right\}=\exp \left\{\frac{-b_{n}^{2}}{2 \sigma^{2} n}\right\} \exp \left\{-\frac{1}{2 \sigma^{2}}\left(\frac{b_{n}^{2}}{K_{n}}-\frac{b_{n}^{2}}{n}\right)\right\} \\
& \sim \operatorname{m} \rightarrow \infty \\
& \exp \left\{\frac{-b_{n}^{2}}{2 \sigma^{2} n}\right\} e^{-\frac{1}{\varepsilon_{n}}}=o\left(\exp \left\{\frac{-b_{n}^{2}}{2 \sigma^{2} n}\right\}\right)
\end{aligned}
$$

и, кроме того,

$$
\int_{1 / n}^{1 / K_{n}} \frac{1}{b_{n}^{2} s^{\frac{7}{2}}} e^{\frac{-b_{n}^{2}}{2 \sigma^{2}} s} d s \leqslant \frac{n}{b_{n}^{2}} \int_{1 / n}^{1 / K_{n}} \frac{1}{s^{\frac{5}{2}}} e^{\frac{-b_{n}^{2}}{2 \sigma^{2}} s} d s=o\left(\int_{K_{n}}^{n} \sqrt{t} e^{-\frac{b_{n}^{2}}{2 t \sigma^{2}}} d t\right) .
$$

Эти соотношения доказывают справедливость (5) и (6).

Далее, применяя экспоненциальное неравенство Чебышёва (см. [11, гл. 9])

$$
\mathrm{P}\left(S_{i}>b_{n}\right) \leqslant e^{-i \Lambda\left(\frac{b_{n}}{i}\right)},
$$

получаем

$$
\sum_{i=1}^{n / 2} \mathrm{P}\left\{S_{i}>b_{n}\right\} \leqslant \sum_{i=1}^{n / 2} e^{-i \Lambda\left(\frac{b_{n}}{i}\right)} .
$$

Оценим снизу функцию $g(x):=x \Lambda\left(\frac{b_{n}}{x}\right)$ для $x \in\left[1, \frac{n}{2}\right]$. Если $x$ таково, что $\frac{b_{n}}{x}$ лежит в некотором достаточно малом интервале $(0, \delta)$, то верно

$$
\frac{d}{d x} g(x)=\Lambda\left(\frac{b_{n}}{x}\right)-\frac{b_{n}}{x} \Lambda^{(1)}\left(\frac{b_{n}}{x}\right)=-\frac{\Lambda^{(2)}(0)}{2 !} \frac{b_{n}^{2}}{x^{2}}+o\left(\frac{b_{n}^{2}}{x^{2}}\right)<0 .
$$


Это значит, что на множестве $\left(\frac{b_{n}}{\delta},+\infty\right)$ функция $g(x)$ убывает с ростом $x$ и

$$
g(x) \geqslant g\left(\frac{n}{2}\right)=\frac{n}{2} \Lambda\left(\frac{2 b_{n}}{n}\right)
$$

для $\frac{b_{n}}{\delta} \leqslant x \leqslant \frac{n}{2}$.

Рассмотрим теперь значения $x$ такие, что $\frac{b_{n}}{x}>\delta$. Поскольку функция $\Lambda(\alpha)$ выпукла вниз и возрастает при $\alpha>0$, прямая, проведенная через начало координат и точку $(\delta ; \Lambda(\delta))$, будет иметь положительный коэффициент наклона $\beta=\frac{\Lambda(\delta)}{\delta}$ и лежать ниже графика $\Lambda(\alpha)$ при $\alpha>\delta$. Стало быть, для $x \in\left(1, \frac{b_{n}}{\delta}\right)$ имеем

$$
g(x) \geqslant \beta x \frac{b_{n}}{x}=\beta b_{n} .
$$

Принимая во внимание, что

$$
\frac{b_{n}^{2} / n}{b_{n}} \underset{n \rightarrow \infty}{\longrightarrow} 0, \quad n \Lambda\left(\frac{b_{n}}{n}\right)=\frac{b_{n}^{2}}{2 \sigma^{2} n}+o\left(\frac{b_{n}^{2}}{n}\right), \quad \frac{n}{2} \Lambda\left(\frac{2 b_{n}}{n}\right)=\frac{b_{n}^{2}}{\sigma^{2} n}+o\left(\frac{b_{n}^{2}}{n}\right),
$$

из соотношений (7) и (8) выводим

$$
\begin{aligned}
\sum_{i=1}^{n / 2} \mathrm{P}\left\{S_{i}>b_{n}\right\} \leqslant & n e^{-\frac{n}{2} \Lambda\left(\frac{2 b_{n}}{n}\right)}+n e^{-\beta b_{n}}=\frac{n^{\frac{5}{2}}}{b_{n}^{3}} e^{-n \Lambda\left(\frac{b_{n}}{n}\right)} e^{3 \ln \left(\frac{b_{n}}{\sqrt{n}}\right)} \\
& \times\left(\exp \left\{-\frac{b_{n}^{2}}{2 \sigma^{2} n}+o\left(\frac{b_{n}^{2}}{n}\right)\right\}+\exp \left\{-\beta b_{n}+\frac{b_{n}^{2}}{2 \sigma^{2} n}+o\left(\frac{b_{n}^{2}}{n}\right)\right\}\right) \\
= & o\left(\frac{n^{\frac{5}{2}}}{b_{n}^{3}} e^{-n \Lambda\left(\frac{b_{n}}{n}\right)}\right) .
\end{aligned}
$$

Осталось найти асимптотику суммы $\sum_{i=n / 2}^{K_{n}}$. При $i \geqslant \frac{n}{2}$ с помощью формулы конечных приращений для достаточно больших $n$ и некоторого числа $\theta_{n} \leqslant \frac{2 b_{n}}{n}$ находим

$$
\left|\lambda\left(\frac{b_{n}}{i}\right)-\lambda\left(\frac{b_{n}}{n}\right)\right|=\left|\lambda^{\prime}\left(\theta_{n}\right)\right|\left(\frac{b_{n}}{i}-\frac{b_{n}}{n}\right) \leqslant C_{1}\left(\frac{b_{n}}{n}\right)^{3} \frac{n-i}{i}
$$

и, следовательно,

$$
\left|i \lambda\left(\frac{b_{n}}{i}\right)-n \lambda\left(\frac{b_{n}}{n}\right)\right| \leqslant i\left|\lambda\left(\frac{b_{n}}{i}\right)-\lambda\left(\frac{b_{n}}{n}\right)\right|+(n-i)\left|\lambda\left(\frac{b_{n}}{n}\right)\right| \leqslant C_{2}\left(\frac{b_{n}}{n}\right)^{3}(n-i) .
$$

Тогда из (2) для достаточно больших $n$ получаем

$$
\begin{aligned}
& \frac{\mathrm{P}\left(S_{i}>b_{n}\right)}{\mathrm{P}\left(S_{n}>b_{n}\right)} \leqslant \frac{1+1 / 2}{1-1 / 2} \sqrt{\frac{i}{n}} \frac{e^{-i \Lambda\left(b_{n} / i\right)}}{e^{-n \Lambda\left(b_{n} / n\right)}} \\
& \leqslant C \exp \left\{-C_{1}\left(\frac{b_{n}}{n}\right)^{2}(n-i)+C_{2}\left(\frac{b_{n}}{n}\right)^{3}(n-i)\right\}, \\
& \sum_{i=n / 2}^{K_{n}} \mathrm{P}\left\{S_{i}>b_{n}\right\} \\
& \leqslant C \mathrm{P}\left(S_{n}>b_{n}\right) \sum_{i=n / 2}^{K_{n}} \exp \left\{-C_{1}\left(\frac{b_{n}}{n}\right)^{2}(n-i)+C_{2}\left(\frac{b_{n}}{n}\right)^{3}(n-i)\right\}
\end{aligned}
$$




$$
\begin{aligned}
& \leqslant C \mathrm{P}\left(S_{n}>b_{n}\right) \sum_{i=n / 2}^{K_{n}} \exp \left\{-C_{3} \frac{b_{n}^{2}}{n^{2}}(n-i)\right\} \\
& \underset{n \rightarrow \infty}{\sim} C \mathrm{P}\left(S_{n}>b_{n}\right) \int_{n / 2}^{K_{n}} \exp \left\{-C_{3} \frac{b_{n}^{2}}{n^{2}}(n-t)\right\} d t \\
& =C \mathrm{P}\left(S_{n}>b_{n}\right) \int_{n-K_{n}}^{n / 2} \exp \left\{-C_{3} \frac{b_{n}^{2}}{n^{2}} s\right\} d s \\
& =O\left(\mathrm{P}\left(S_{n}>b_{n}\right) \frac{n^{2}}{b_{n}^{2}} e^{-C_{3} \frac{b_{n}^{2}}{n^{2}}\left(n-K_{n}\right)}\right)=O\left(\frac{n^{\frac{5}{2}}}{b_{n}^{3}} e^{-\frac{C_{4}}{\varepsilon_{n}}} e^{-n \Lambda\left(\frac{b_{n}}{n}\right)}\right) .
\end{aligned}
$$

Из соотношений (6), (9) и (10) получаем утверждение теоремы 1.

\section{§ 3. Доказательство теоремы 2}

Обозначим $L_{\delta}=\{s:|s|<1,|s-1|<\delta\}$. Тогда при любом малом $\delta>0$ имеем

$$
\mathrm{E} T_{n}(b)=\frac{1}{2 \pi i} \int_{|s|=1,|s-1| \geqslant \delta} \frac{r(s)}{s^{n+1}} d s+\frac{1}{2 \pi i} \int_{\Gamma} \frac{r(s)}{s^{n+1}} d s=I_{1}+I_{2},
$$

где $\Gamma$ - лежащий в $L_{\delta}$ контур, полученный из дуги $|s|=1,|s-1|<\delta$ искривлением вблизи единицы внутрь круга $|s|<1$. Смысл такого искривления контура будет объяснен ниже. Первый интеграл в этом соотношении определен корректно, так как функция $r(s)$ допускает аналитическое продолжение в некоторую окрестность дуги $|s|=1,|s-1| \geqslant \delta$, что также будет показано далее в лемме 2 .

Оценим интегралы $I_{1}$ и $I_{2}$. Начнем с оценки $I_{1}$. Для этого нам потребуется ряд предварительных результатов.

В дальнейшем будем использовать следующее обозначение: для любого борелевского множества $B \in \mathbb{B}(\mathbb{R})$

$$
\left[\int_{-\infty}^{+\infty} e^{\lambda x} d V(x)\right]^{B}=\int_{B} e^{\lambda x} d V(x), \quad \text { где } \quad \int_{-\infty}^{+\infty}|d V(x)|<\infty, \quad \operatorname{Re} \lambda=0 .
$$

Лемма 1. Пусть функиия $Q(z, \lambda)$ дифферениируема по z в некоторой области $U$ и выполнено

$$
\begin{array}{cc}
Q(z, \lambda)=\int_{-\infty}^{+\infty} e^{\lambda t} d G_{z}(t), \quad \operatorname{Re} \lambda=0 \\
\frac{\partial}{\partial z} Q(z, \lambda)=\int_{-\infty}^{+\infty} e^{\lambda t} d F_{z}(t), & \operatorname{Re} \lambda=0
\end{array}
$$

где $G_{z}$ и $F_{z}$ - функции ограниченной вариащии. Тогда для любых $B \in \mathbb{B}(\mathbb{R})$, $z \in U$

$$
\frac{\partial}{\partial z}[Q(z, \lambda)]^{B}=\left[\frac{\partial}{\partial z} Q(z, \lambda)\right]^{B}
$$


ДокАЗАТЕЛЬСтво. Положим

$$
F_{z}^{h}(t)=\frac{G_{z+h}(t)-G_{z}(t)}{h} .
$$

Функции $F_{z}^{h}(t)$ имеют ограниченную вариацию, и

$$
\frac{\partial}{\partial z} Q(z, \lambda)=\lim _{h \rightarrow 0} \int_{-\infty}^{+\infty} e^{\lambda t} d F_{z}^{h}(t)=\int_{-\infty}^{+\infty} e^{\lambda t} d F_{z}(t), \quad \operatorname{Re} \lambda=0 .
$$

Отсюда получаем, что $F_{z}^{h}(t) \underset{h \rightarrow 0}{\longrightarrow} F_{z}(t)$ в каждой точке $t$, являющейся точкой непрерывности $F_{z}(t)$. Следовательно, для любых $B \in \mathbb{B}(\mathbb{R})$ и $z \in U$ выполнено

$$
\frac{\partial}{\partial z}[Q(z, \lambda)]^{B}=\lim _{h \rightarrow 0} \int_{B} e^{\lambda t} d F_{z}^{h}(t)=\int_{B} e^{\lambda t} d F_{z}(t)=\left[\frac{\partial}{\partial z} Q(z, \lambda)\right]^{B}, \quad \operatorname{Re} \lambda=0,
$$

что и требовалось доказать.

Лемма 2. Пусть выполнены условия теоремы 2. Тогда для любого $\delta>0$ существует число $\varepsilon>0$ такое, что

$$
I_{1}=\frac{1}{2 \pi i} \int_{|s|=1,|s-1| \geqslant \delta} \frac{r(s)}{s^{n+1}} d s=O\left(e^{-\varepsilon b}\right)
$$

npu $b \rightarrow \infty$.

ДокАЗАТЕЛЬСтво. Пусть $\varphi(\lambda)=\mathrm{E} e^{\lambda X_{1}}$. Мы будем использовать факторизацию

$$
1-z \varphi(\lambda)=R_{+}(z, \lambda) R_{-}(z, \lambda) R_{0}(z), \quad|z|<1, \quad \operatorname{Re} \lambda=0,
$$

В которой

$$
\begin{gathered}
R_{0}(z)=\exp \left\{-\sum_{n=1}^{\infty} \frac{z^{n}}{n} \mathrm{P}\left(S_{n}=0\right)\right\}, \\
R_{-}(z, \lambda)=\exp \left\{-\sum_{n=1}^{\infty} \frac{z^{n}}{n} \mathrm{E}\left(e^{\lambda S_{n}} ; S_{n}<0\right)\right\}, \\
R_{+}(z, \lambda)=\exp \left\{-\sum_{n=1}^{\infty} \frac{z^{n}}{n} \mathrm{E}\left(e^{\lambda S_{n}} ; S_{n}>0\right)\right\} .
\end{gathered}
$$

Известны также и другие представления для компонент факторизации. Пусть

$$
\eta_{-}=\inf \left\{n \geqslant 1: S_{n}<0\right\}, \quad \eta_{+}=\inf \left\{n \geqslant 1: S_{n}>0\right\}, \quad \chi_{ \pm}=S_{\eta_{ \pm}} .
$$

Тогда

$$
R_{ \pm}(z, \lambda)=1-\mathrm{E}\left(z^{\eta_{ \pm}} \exp \left\{\lambda \chi_{ \pm}\right\}\right), \quad \operatorname{Re} \lambda=0, \quad|z| \leqslant 1 .
$$

При выполнении условий теоремы 2 функция $1-z \varphi(\lambda)$ и компоненты факторизации $R_{ \pm}(z, \lambda)$ обладают рядом свойств, которые будут нами использоваться в дальнейшем и которые подробно изложены в [6]. Приведем основные из них. 
Как следует из условия $(\mathrm{C})$, при некотором $\delta_{1}>0$ функция $1-z \varphi(\lambda)$ (по переменной $\lambda$ ) имеет ровно два вещественных нуля $\lambda_{ \pm}(z), z \in\left[1-\delta_{1}, 1\right]$; здесь $\lambda_{-}(z) \leqslant 0 \leqslant \lambda_{+}(z)$ и $\lambda_{ \pm}(1)=0$. Это легко понять, поскольку график выпуклой вниз функции $\varphi(\lambda)$ дважды пересекает горизонтальную прямую на уровне $1 / z \geqslant 1$. Функции $\lambda_{ \pm}(z)$ являются ветвями двузначной функции с точкой ветвления $z=1$. В некоторой окрестности единицы, разрезанной по лучу $z \geqslant 1$, имеют место разложения

$$
\lambda_{ \pm}(z)=\mp \psi_{1} i(z-1)^{1 / 2}-\psi_{2}(z-1) \mp \cdots
$$

(см. [6]), значения коэффициентов $\psi_{1}, \psi_{2}$ указаны в формулировке теоремы 2. Здесь функция $t=i(z-1)^{1 / 2}$ переводит полуплоскость $\operatorname{Im} z>0$ в область $\pi / 2<$ $\arg t<\pi$. Функции $\lambda_{ \pm}(z)$ могут быть аналитически продолжены в некоторую $\delta$-окрестность отрезка $\left[1-\delta_{1}, 1\right]$ с разрезом по лучу $z \geqslant 1$. При этом $\lambda_{ \pm}(z)$ по-прежнему остаются нулями функции $1-z \varphi(\lambda)$. Функции $\lambda_{ \pm}(z)$ являются также нулями компонент факторизации: $R_{ \pm}\left(z, \lambda_{ \pm}(z)\right)=0$.

При выполнении условия (C) введенная факторизация справедлива в более широкой области $|\operatorname{Re} \lambda| \leqslant \beta$. Функция $R_{+}(z, \lambda)$ аналитична по совокупности переменных $(z, \lambda)$ на множестве $|z|<1, \operatorname{Re} \lambda<\beta$, на котором она является преобразованием Лапласа-Стилтьеса (по переменной $\lambda$ ) зависящей от $z$ функции ограниченной вариации, все изменение которой сосредоточено на $[0, \infty)$. Аналогичными свойствами при $\operatorname{Re} \lambda>-\beta$ обладает функция $R_{-}(z, \lambda)$.

Известно также (см. [6, теорема 3 и ее следствия]), что в окрестности точки $z=1$ справедливо представление

$$
R_{+}(z, \lambda)=\frac{\lambda-\lambda_{+}(z)}{\lambda-1} W_{+}(z, \lambda)
$$

в котором функции $W_{+}^{ \pm 1}(z, \lambda)$ аналитичны по совокупности переменных в окрестности точки $z=1$ и при $\operatorname{Re} \lambda \leqslant \varepsilon, \varepsilon>0$. Аналог этого свойства (с очевидными изменениями) имеет место и для $R_{-}(z, \lambda)$. Соотношение $(12)$ вместе с (11) позволяет определять поведение компоненты факторизации $R_{+}(z, \lambda)$ в окрестности точки $z=1$.

Везде далее, где будет встречаться производная от компоненты факторизации, предполагаем, что производная берется по второму аргументу $\lambda$ :

$$
R_{ \pm}^{\prime}(z, \lambda):=\frac{\partial R_{ \pm}(z, \lambda)}{\partial \lambda} .
$$

В [9] установлено, что для $|s|<1,|s v|<1, \operatorname{Re} \lambda=0$

$$
\sum_{k=0}^{\infty} s^{k} \mathrm{E} v^{T_{k}}=\sum_{k=0}^{\infty} s^{k} \sum_{l=0}^{k} v^{l} \mathrm{P}\left(T_{k}=l\right)=\frac{1}{1-s}-H(s, v),
$$

где

$$
T_{k}=T_{k}(b), \quad H(s, v)=\frac{R_{+}(s, 0)}{(1-s) R_{+}(s v, 0)}\left[\frac{R_{+}(s v, \lambda)}{R_{+}(s, \lambda)}\right]_{\lambda=0}^{(b, \infty)}
$$


Дифференцируя (13) по $v$ и полагая $v=1$, получаем

$$
r(s)=-\left.\frac{\partial H(s, v)}{\partial v}\right|_{v=1}
$$

Из леммы 1 следует

$$
-\left.\frac{\partial H(s, v)}{\partial v}\right|_{v=1}=\frac{s}{s-1}\left(\left[\frac{\left.\frac{\partial R_{+}(z, \lambda)}{\partial z}\right|_{z=s v}}{R_{+}(s, \lambda)}\right]_{\substack{\lambda=0 \\ v=1}}^{(b, \infty)}-\frac{\left.\frac{\partial R_{+}(z, 0)}{\partial z}\right|_{z=s}}{R_{+}(s, 0)}\left[\frac{R_{+}(s v, \lambda)}{R_{+}(s, \lambda)}\right]_{\substack{\lambda=0 \\ v=1}}^{(b, \infty)}\right) .
$$

Существование производной по $z$ следует из аналитичности $R_{+}(z, \lambda)$ в некоторой окрестности дуги $\{|z|=1,|z-1| \geqslant \delta\}$, этот факт содержится в [6, теорема 2].

Для указанных значений переменной $z$ функция $1-z \varphi(\lambda)$, а вместе с ней и $R_{+}(z, \lambda)$ не имеют нулей в полосе $0 \leqslant \operatorname{Re} \lambda \leqslant \varepsilon$ для некоторого $\varepsilon>0$. По этой причине для любого $\delta>0$ найдется число $\varepsilon>0$ такое, что при $|s|=1$, $|s-1| \geqslant \delta$ имеют место представления

$$
\begin{gathered}
\frac{R_{+}(s v, \lambda)}{R_{+}(s, \lambda)}=\int_{0}^{\infty} e^{\lambda t} d G_{s, v}(t), \quad \operatorname{Re} \lambda \leqslant \varepsilon, \quad \int_{0}^{\infty} e^{\varepsilon t}\left|d G_{s, v}(t)\right| \leqslant C<\infty \\
\left.R_{+}^{-1}(s, \lambda) \frac{\partial R_{+}(z, \lambda)}{\partial z}\right|_{z=s v}=\int_{0}^{\infty} e^{\lambda t} d F_{s, v}(t), \quad \operatorname{Re} \lambda \leqslant \varepsilon \\
\int_{0}^{\infty} e^{\varepsilon t}\left|d F_{s, v}(t)\right| \leqslant C<\infty
\end{gathered}
$$

Тогда

$$
\begin{aligned}
& \left|\frac{\partial H(s, v)}{\partial v}\right|_{v=1}\left|\leqslant C_{1} \int_{(b, \infty)}\right| d G_{s, 1}(t)\left|+C_{2} \int_{(b, \infty)}\right| d F_{s, 1}(t) \mid \\
& =C_{1} e^{-\varepsilon b} \int_{(b, \infty)} e^{\varepsilon b}\left|d G_{s, 1}(t)\right|+C_{2} e^{-\varepsilon b} \int_{(b, \infty)} e^{\varepsilon b}\left|d F_{s, 1}(t)\right| \\
& \leqslant C_{1} e^{-\varepsilon b} \int_{(b, \infty)} e^{\varepsilon y}\left|d G_{s, 1}(t)\right|+C_{2} e^{-\varepsilon b} \int_{(b, \infty)} e^{\varepsilon y}\left|d F_{s, 1}(t)\right| \leqslant C_{3} e^{-\varepsilon b} .
\end{aligned}
$$

В итоге имеем

$$
\begin{aligned}
I_{1} & =\frac{1}{2 \pi i} \int_{|s|=1,|s-1| \geqslant \delta} \frac{r(s)}{s^{n+1}} d s \\
& =-\left.\frac{1}{2 \pi i} \int_{|s|=1,|s-1| \geqslant \delta} \frac{1}{s^{n+1}} \frac{\partial H(s, v)}{\partial v}\right|_{v=1} d s=O\left(e^{-\varepsilon b}\right) .
\end{aligned}
$$

Лемма доказана.

Вернемся к доказательству теоремы 2. Будем оценивать интеграл $I_{2}$, полагая теперь, что $b=b_{n}$ удовлетворяет условию теоремы. Асимптотический анализ будет осуществляться с помощью метода перевала, а точнее с помощью той его модификации, когда в окрестности точки перевала находится близкий полюс. 
Известно [10], что найдутся числа $\delta>0$ и $\varepsilon>0$ такие, что при $b_{n} \underset{n \rightarrow \infty}{\longrightarrow} \infty$ равномерно по $s \in L_{\delta}$ имеет место равенство

$$
\sum_{k=1}^{\infty} s^{k} \mathrm{E} T_{k}\left(b_{n}\right)=\frac{v(s) e^{-\lambda_{+}(s) b_{n}} A(s)}{s-1}+\frac{O\left(e^{-\varepsilon b_{n}}\right)}{s-1},
$$

где

$$
\begin{gathered}
v(s)=-\frac{R_{+}(s, 0)}{\lambda_{+}(s) R_{+}^{\prime}\left(s, \lambda_{+}(s)\right)}, \\
A(s)=\left.\frac{\partial}{\partial v} \frac{R_{+}\left(s v, \lambda_{+}(s)\right)}{R_{+}(s v, 0)}\right|_{v=1}=\left.s R_{+}^{-1}(s, 0) \frac{\partial R_{+}}{\partial z}(z, \lambda)\right|_{\substack{z=s \\
\lambda=\lambda_{+}(s)}} .
\end{gathered}
$$

Напомним, что $\lambda_{+}(s)-$ нуль функции $R_{+}(s, \lambda)$. Сначала рассмотрим первое слагаемое в правой части (14). Из (12) следует

$$
\left.\frac{\partial R_{+}(z, \lambda)}{\partial z}\right|_{\substack{z=s \\ \lambda=\lambda_{+}(s)}}=\frac{-\lambda_{+}^{\prime}(s)}{\lambda_{+}(s)-1} W\left(s, \lambda_{+}(s)\right), \quad R_{+}^{\prime}\left(s, \lambda_{+}(s)\right)=\frac{W\left(s, \lambda_{+}(s)\right)}{\lambda_{+}(s)-1},
$$

а отношение этих функций равно $-\lambda_{+}^{\prime}(s)$. Таким образом, первое слагаемое из правой части (14) принимает вид

$$
\frac{s}{s-1} \frac{\lambda_{+}^{\prime}(s)}{\lambda_{+}(s)} e^{-\lambda_{+}(s) b_{n}} .
$$

Рассмотрим теперь интеграл $I_{2}$. Как уже отмечалось, интегрирование в нем проводится вдоль контура $\Gamma$, лежащего в $L_{\delta}$ и полученного искривлением дуги $\{|s|=1,|s-1|<\delta\}$ внутрь круга $|s|<1$. Искривление контура потребовалось в связи с наличием особенности подынтегральной функции в точке $s=1$. Интегрируя главную часть асимптотического представления (14), получаем

$$
\frac{1}{2 \pi i} \int_{\Gamma} \frac{1}{s^{n+1}} \frac{s}{s-1} \frac{\lambda_{+}^{\prime}(s)}{\lambda_{+}(s)} e^{-\lambda_{+}(s) b_{n}} d s=\frac{1}{2 \pi i} \int_{\Gamma} \frac{1}{s-1} \frac{\lambda_{+}^{\prime}(s)}{\lambda_{+}(s)} e^{n \hat{h}_{n}(s)} d s,
$$

где $\hat{h}_{n}(s)=-\frac{b_{n}}{n} \lambda_{+}(s)-\ln (s)$. Воспользуемся разложением (11). Пусть $G-$ некоторая окрестность $Г$, отделенная от единицы. Применив почленное дифференцирование, для $s \in G$ можно записать разложение

$$
\mu_{+}(s):=\lambda_{+}^{\prime}(s)=-\frac{\psi_{1}}{2} i(s-1)^{-\frac{1}{2}}-\psi_{2}-\cdots .
$$

Покажем возможность такого дифференцирования. Пусть $P$ и $Q$ - такие степенные ряды, что в $G$ выполнено

$$
\begin{gathered}
\lambda_{+}(s)=P\left(i(s-1)^{\frac{1}{2}}\right), \\
\mu_{+}(s)=-\frac{\psi_{1}}{2} i(s-1)^{-\frac{1}{2}}+Q\left(i(s-1)^{\frac{1}{2}}\right) .
\end{gathered}
$$

Ясно, что $P$ и $Q$ имеют одинаковые радиусы сходимости. Тогда из сходимости ряда $P\left(i(s-1)^{1 / 2}\right)$ в некоторой точке $s \in G$ следует сходимость (равномерная 
в $G)$ ряда $Q\left(i(s-1)^{1 / 2}\right)$. Далее, так как функция $-\frac{\psi_{1}}{2} i(s-1)^{-1 / 2}$ аналитична в области $G$, то и $\mu_{+}(s)$ аналитична в $G$ и является в ней производной $\lambda_{+}(s)$.

Сделаем замену переменных $t=i(s-1)^{1 / 2}$; тогда

$$
d s=-2 t d t
$$

и пусть

$$
\lambda(t)=-\psi_{1} t-\psi_{2} t^{2}+\cdots, \quad \mu(t)=\frac{\psi_{1}}{2} \frac{1}{t}-\psi_{2}+\cdots
$$

Тогда

$$
\frac{1}{2 \pi i} \int_{\Gamma} \frac{1}{s-1} \frac{\mu_{+}(s)}{\lambda_{+}(s)} e^{n \hat{h}_{n}(s)} d s=\frac{1}{2 \pi i} \int_{\Gamma_{1}} \frac{2 \mu(t)}{t \lambda(t)} e^{n h_{n}(t)} d t=\frac{1}{2 \pi i} \int_{\Gamma_{1}} \frac{g(t) e^{n h(t)}}{t^{3}} d t
$$

где

$$
g(t)=\frac{2 t^{2} \mu(t)}{\lambda(t)}
$$

- аналитическая по $t$ в окрестности нуля функция, $\Gamma_{1}$ - образ контура $\Gamma$ в плоскости переменной $t$, а $h_{n}(t)=\hat{h}_{n}(s)$.

Напомним, что $\tau_{n}=\frac{b_{n}}{n}$. Тогда, как легко видеть,

$$
h_{n}(t)=\tau_{n}\left(\psi_{1} t+\psi_{2} t^{2}+\cdots\right)+\left(t^{2}+\frac{t^{4}}{2}+\cdots\right) .
$$

Точка перевала $t_{n}$ является решением уравнения

$$
h_{n}^{\prime}(t)=\tau_{n}\left(\psi_{1}+2 \psi_{2} t+3 \psi_{3} t^{2}+\cdots\right)+2\left(t+t^{3}+\cdots\right)=0,
$$

поэтому (см. [5])

$$
\begin{gathered}
t_{n}=-\frac{\tau_{n} \psi_{1}}{2}\left(1-\tau_{n} \psi_{2}\right)+O\left(\tau_{n}^{3}\right), \\
d\left(\tau_{n}\right):=h_{n}\left(t_{n}\right)=-\frac{\tau_{n}^{2} \psi_{1}^{2}}{4}+\frac{3 \tau_{n}^{3} \psi_{1}^{3} \psi_{2}}{4}+O\left(\tau_{n}^{4}\right), \\
\nu_{n}=\frac{h_{n}^{\prime \prime}\left(t_{n}\right)}{2}=1+\psi_{2} \tau_{n}+O\left(\tau_{n}^{2}\right) .
\end{gathered}
$$

Поскольку $h_{n}^{\prime}\left(t_{n}\right)=0$, кривые $\operatorname{Re} h_{n}(t)=h_{n}\left(t_{n}\right)$ делят окрестность точки $t_{n}$ на четыре сектора, в которых поочередно $\operatorname{Re} h_{n}(t)<h_{n}\left(t_{n}\right)$ и $\operatorname{Re} h_{n}(t)>h_{n}\left(t_{n}\right)$. Концы контура $\Gamma_{1}$ при достаточно малых значениях $\tau_{n}$ лежат внутри секторов $\operatorname{Re} h_{n}(t)<h_{n}\left(t_{n}\right)$. Изменим контур $\Gamma_{1}$ так, чтобы, оставаясь внутри секторов $\operatorname{Re} h_{n}(t)<h_{n}\left(t_{n}\right)$, он проходил через точку $t_{n}$. Ясно, что при некотором $\delta>0$ интеграл (15) с точностью до экспоненциально малой величины принимает вид

$$
\frac{1}{2 \pi i} \int_{t_{n}-i \delta}^{t_{n}+i \delta} \frac{g(t) e^{n h_{n}(t)}}{t^{3}} d t=\frac{1}{2 \pi} \int_{-\delta}^{\delta} \frac{g\left(t_{n}+i u\right) e^{n h_{n}\left(t_{n}+i u\right)}}{\left(t_{n}+i u\right)^{3}} d u .
$$


Согласно условиям теоремы существует число $\varepsilon>0$ такое, что

$$
\frac{n^{\varepsilon+1 / 2}}{b_{n}} \underset{n \rightarrow \infty}{\longrightarrow} 0
$$

Без ограничения общности можно считать, что $\varepsilon<\frac{1}{6}$. Положим $\eta_{n}=n^{\varepsilon-1 / 2}$; тогда $\eta_{n}=o\left(n^{-1 / 3}\right)$ при $n \rightarrow \infty$.

Покажем что интегрирование по множеству $\left(-\delta,-\eta_{n}\right) \cup\left(\eta_{n}, \delta\right)$ вносит пренебрежимо малый вклад в интеграл (17). Из определения функции $h_{n}$ ясно, что в некоторой окрестности нуля можно воспользоваться приближением Тейлора

$$
h_{n}\left(t_{n}+i u\right)=h_{n}\left(t_{n}\right)-\frac{u^{2}}{2} h_{n}^{\prime \prime}\left(t_{n}\right)+R\left(t_{n}, u\right)
$$

в котором равномерно по $t_{n}$ имеет место оценка $\left|R\left(t_{n}, u\right)\right| \leqslant H|u|^{3}$ при некоторой постоянной $H>0$. По этой причине для значений переменной интегрирования $u$ из $\left(-\delta,-\eta_{n}\right) \cup\left(\eta_{n}, \delta\right)$ при достаточно малом $\delta$ выполнено

$$
\begin{aligned}
& \operatorname{Re}\left\{n h_{n}\left(t_{n}+i u\right)\right\} \leqslant n\left(h_{n}\left(t_{n}\right)-\frac{u^{2}}{2} h_{n}^{\prime \prime}\left(t_{n}\right)+H|u|^{3}\right) \\
& \leqslant n h_{n}\left(t_{n}\right)-\frac{h_{n}^{\prime \prime}\left(t_{n}\right)}{4} n \eta_{n}^{2}-n u^{2}\left(\frac{h_{n}^{\prime \prime}\left(t_{n}\right)}{4}-H \delta\right) \leqslant n h_{n}\left(t_{n}\right)-C n^{2 \varepsilon} .
\end{aligned}
$$

Кроме того,

$$
n h_{n}\left(t_{n}\right) \sim-C_{1} \frac{b_{n}^{2}}{n}, \quad n^{2 \varepsilon}=o\left(\frac{b_{n}^{2}}{n}\right), \quad\left|t_{n}+i u\right| \geqslant\left|t_{n}\right| \sim C_{2} \frac{b_{n}}{n} \geqslant C_{2} n^{-\frac{1}{2}},
$$

поэтому

$$
\left|\int_{\eta_{n}<|u|<\delta} \frac{g\left(t_{n}+i u\right) e^{n h_{n}\left(t_{n}+i u\right)}}{\left(t_{n}+i u\right)^{3}} d u\right| \leqslant C_{3} n^{\frac{3}{2}} e^{n h_{n}\left(t_{n}\right)-C_{4} n^{2 \varepsilon}} \leqslant C_{5} e^{n h_{n}\left(t_{n}\right)-C n^{2 \varepsilon}} .
$$

Рассмотрим теперь интеграл по множеству $\left\{|u| \leqslant \eta_{n}\right\}$. Из соотношения $t_{n} \sim-\frac{\tau_{n} \psi_{1}}{2}$ следует $\eta_{n}=o\left(t_{n}\right)$. Поэтому для достаточно больших $n$ круг радиуса $\eta_{n}$ с центром в точке $t_{n}$ не содержит нуля, а значит, функция $\frac{1}{\left(t_{n}+i u\right)^{3}}$ может быть разложена в этом круге в ряд Тейлора:

$$
\frac{1}{\left(t_{n}+i u\right)^{3}}=\sum_{k=0}^{\infty} \frac{f_{k}}{t_{n}^{k+3}}(i u)^{k}, \quad f_{k}=\frac{(-1)^{k}(k+1)(k+2)}{2} .
$$

Кроме того, будем использовать разложение

$$
h_{n}\left(t_{n}+i u\right)-h_{n}\left(t_{n}\right)+\frac{u^{2}}{2} h_{n}^{\prime \prime}\left(t_{n}\right)=-\frac{i u^{3}}{3 !} h_{n}^{\prime \prime \prime}\left(t_{n}\right)+\cdots
$$


Заметив, что при достаточно больших значениях $n$ имеет место неравенство $|u|<\delta n^{-1 / 3}$, выпишем разложение

$$
\begin{aligned}
& \frac{g\left(t_{n}+i u\right)}{\left(t_{n}+i u\right)^{3}} \exp \left\{n\left(-\frac{i u^{3}}{3 !} h_{n}^{\prime \prime \prime}\left(t_{n}\right)+\cdots\right)\right\} \\
& \quad=\frac{1}{t_{n}^{3}}\left(\sum_{k=0}^{\infty} \frac{(-i)^{k}(k+1)(k+2) u^{k}}{2 t_{n}^{k}}\right)\left(\sum_{l, m=0}^{\infty} c_{l m} u^{l}\left(n u^{3}\right)^{m}\right) \\
& =\frac{1}{t_{n}^{3}}\left(\sum_{k=0}^{\infty} \frac{(-i)^{k}(k+1)(k+2) u^{k}}{2 t_{n}^{k}}\right)\left(\sum_{\substack{l+m \leqslant N \\
l \geqslant 0, m \geqslant 0}} c_{l m} u^{l}\left(n u^{3}\right)^{m}+\Delta_{N}\left(u, n u^{3}\right)\right),
\end{aligned}
$$

где

$$
\Delta_{N}\left(u, n u^{3}\right)=O\left(|u|^{N+1}\right)+O\left(\left|n u^{3}\right|^{N+1}\right) .
$$

Эта оценка верна для любого $N \geqslant 0$ (см. [13]). Из того, что $t_{n} \underset{n \rightarrow \infty}{\sim} C \tau_{n}$, получаем

$$
\left|\frac{u}{t_{n}}\right| \leqslant C \frac{n}{b_{n}} \frac{n^{\varepsilon}}{\sqrt{n}} \underset{n \rightarrow \infty}{\longrightarrow} 0 .
$$

Следовательно, имеем

$$
\sum_{k=0}^{\infty} \frac{(-i)^{k}(k+1)(k+2)}{2} \frac{u^{k}}{t_{n}^{k}} \underset{n \rightarrow \infty}{\longrightarrow} 1
$$

откуда

$$
\left(\sum_{k=0}^{\infty} \frac{(-i)^{k}(k+1)(k+2) u^{k}}{2 t_{n}^{k}}\right) \Delta_{N}\left(u, n u^{3}\right)=O\left(|u|^{N+1}\right)+O\left(\left|n u^{3}\right|^{N+1}\right) .
$$

Имеем далее

$$
\begin{aligned}
& \sum_{k=0}^{\infty} \frac{(-i)^{k}(k+1)(k+2) u^{k}}{2 t_{n}^{k}} \sum_{\substack{l+m \leqslant N \\
l \geqslant 0, m \geqslant 0}} c_{l m} u^{l}\left(n u^{3}\right)^{m} \\
& =\sum_{\substack{l+m+k \leqslant N \\
l \geqslant 0, m \geqslant 0, k \geqslant 0}} \frac{c_{l m k}}{t_{n}^{k}} u^{l+k}\left(n u^{3}\right)^{m}+\sum_{\substack{l+m \leqslant N \\
l+m+k>N \\
l \geqslant 0, m \geqslant 0, k \geqslant 0}} c_{l m k} u^{l}\left(n u^{3}\right)^{m} \frac{u^{k}}{t_{n}^{k}}
\end{aligned}
$$

где $c_{l m k}=i^{k} f_{k} c_{l m}$.

Разобьем второе слагаемое в (19) на две суммы:

$$
\sum_{\substack{l+m \leqslant N \\ l+m+k>N \\ l \geqslant 0, m \geqslant 0, k \geqslant 0}} c_{l m k} u^{l}\left(n u^{3}\right)^{m} \frac{u^{k}}{t_{n}^{k}}=\sum_{\substack{l+m \leqslant N \\ l+m+k>N \\ l \geqslant 0, m \geqslant 0,0 \leqslant k \leqslant N}}+\sum_{\substack{l+m \leqslant N \\ l \geqslant 0, m \geqslant 0, k \geqslant N+1}} .
$$


Оценим первую из них:

$$
\left|\sum_{\substack{l+m \leqslant N \\ l+m+k>N \\ l \geqslant 0, m \geqslant 0,0 \leqslant k \leqslant N}} c_{l m k} \frac{u^{k+l}\left(n u^{3}\right)^{m}}{t_{n}^{k}}\right|=O\left(\frac{|u|^{N+1}}{t_{n}^{N+1}}\right)+O\left(\frac{\left|u\left(n u^{3}\right)^{N}\right|}{t_{n}^{N+1}}\right) .
$$

Поскольку $\eta_{n} \underset{n \rightarrow \infty}{\longrightarrow} 0$ и $n \eta_{n}^{3} \underset{n \rightarrow \infty}{\longrightarrow} 0$, имеем оценку для второй суммы

$$
\begin{aligned}
& \left|\sum_{\substack{l+m \leqslant N \\
l \geqslant 0, m \geqslant 0 \\
k \geqslant N+1}} c_{l m k} u^{l}\left(n u^{3}\right)^{m} \frac{u^{k}}{t_{n}^{k}}\right|=\left|\sum_{k \geqslant N+1} \frac{f_{k}(i u)^{k}}{t_{n}^{k}}\left(\sum_{\substack{l+m \leqslant N \\
l \geqslant 0, m \geqslant 0}} c_{l m} u^{l}\left(n u^{3}\right)^{m}\right)\right| \\
& \quad \leqslant C \sum_{k \geqslant N+1} f_{k} \frac{|u|^{k}}{t_{n}^{k}}=C \frac{|u|^{N+1}}{t_{n}^{N+1}} \sum_{k \geqslant 0} f_{k+(N+1)} \frac{|u|^{k}}{t_{n}^{k}}=O\left(\frac{|u|^{N+1}}{t_{n}^{N+1}}\right) .
\end{aligned}
$$

Таким образом, правая часть (18) равна

$$
\sum_{\substack{l+m+k \leqslant N \\ l \geqslant 0, m \geqslant 0, k \geqslant 0}} \frac{c_{l m k}}{t_{n}^{k}} u^{l+k}\left(n u^{3}\right)^{m}+\frac{\hat{\Delta}_{N}(u)}{t_{n}^{N+1}}
$$

где $\hat{\Delta}_{N}\left(u, n u^{3}\right)=O\left(|u|^{N+1}\right)+O\left(\left|n u^{3}\right|^{N+1}\right)$. Поэтому интеграл (17) примет вид

$$
\begin{aligned}
& \frac{1}{2 \pi} e^{n h_{n}\left(t_{n}\right)} \sum_{\substack{l+m+k \leqslant N \\
l \geqslant 0, m \geqslant 0, k \geqslant 0}} \frac{c_{l m k}}{t_{n}^{k}} n^{m} \int_{-\delta}^{\delta} u^{l+3 m+k} e^{-n u^{2} \nu_{n}} d u \\
& +\frac{1}{2 \pi} \frac{e^{n h_{n}\left(t_{n}\right)}}{t_{n}^{N+1}} \int_{-\delta}^{\delta} \hat{\Delta}_{N}\left(u, n u^{3}\right) e^{-n u^{2} \nu_{n}} d u+e^{n h_{n}\left(t_{n}\right)} e^{-C n^{2 \varepsilon}+O\left(n \eta_{n}^{3}\right)} .
\end{aligned}
$$

Ясно, что замена интегралов $\int_{-\delta}^{\delta}$ в $(20)$ на интегралы вида $\int_{-\infty}^{+\infty}$ добавит погрешность порядка $O\left(e^{-\varepsilon n}\right)$ при некотором $\varepsilon$. Если число $l+3 m+k$ четно, то получаем

$$
\int_{-\infty}^{+\infty} u^{l+3 m+k} e^{-n u^{2} \nu_{n}} d u=\left(n \nu_{n}\right)^{-\frac{1}{2}(l+3 m+k+1)} \Gamma\left(\frac{l+3 m+k+1}{2}\right) .
$$

Для нечетных $l+3 m+k$ этот интеграл равен нулю. В итоге получаем, что с точностью до экспоненциально малых слагаемых выполнено равенство

$$
\frac{1}{2 \pi} \int_{-\delta}^{\delta} \frac{g\left(t_{n}+i u\right) e^{n h_{n}\left(t_{n}+i u\right)}}{\left(t_{n}+i u\right)^{3}} d u=\frac{e^{n h_{n}\left(t_{n}\right)}}{2 \pi \sqrt{n} t_{n}^{3}}\left(\sum_{k=0}^{2 N-2} \frac{\left(\nu_{n} n\right)^{-\frac{k}{2}}}{t_{n}^{k}} P_{k}(n)+O\left(\frac{n^{-N}}{t_{n}^{2 N}}\right)\right),
$$

где

$$
P_{k}(n)=\sum_{\substack{l+m \leqslant 2 N-k-2 \\ l+m \equiv k(\bmod 2) \\ l \geqslant 0, m \geqslant 0}} c_{l m k} \Gamma\left(\frac{l+3 m+k+1}{2}\right) \nu_{n}^{-\frac{l+3 m}{2}} n^{-\frac{l+m}{2}}
$$


Наконец, остается исследовать вклад остаточного члена из правой части (14). Введенный выше контур $\Gamma$, перешедший после замены $t=i(s-1)^{1 / 2}$ в контур $\Gamma_{1}$, проходит через точку, соответствующую $1-t_{n}^{2}$ в результате этой замены. Поскольку конец Г лежит на единичной окружности, мы можем деформировать его в контур $\Gamma^{\prime}$ так, что расстояние от него до точки $s=1$ станет не менее $t_{n}^{2}$, а до точки $s=0$ не менее $1-t_{n}^{2}$. В результате этих действий интегрирование остаточного члена из (14) приводит к оценке

$$
\begin{aligned}
\left|e^{-\varepsilon b_{n}} \int_{\Gamma} \frac{d s}{(s-1) s^{n+1}}\right| & \leqslant \frac{C e^{-\varepsilon b_{n}}}{t_{n}^{2}\left(1-t_{n}^{2}\right)^{n+1}} \leqslant C e^{-(n+1) \ln \left(1-t_{n}^{2}\right)} e^{-\varepsilon^{\prime} b_{n}} \\
& \leqslant C e^{C^{\prime} b_{n}^{2}(n+1) n^{-2}} e^{-\varepsilon^{\prime} b_{n}}=O\left(e^{-\varepsilon^{\prime \prime} b_{n}}\right) .
\end{aligned}
$$

Теорема 2 доказана.

Выделим главный член полученного разложения. Из (16) и (18) имеем $c_{000}=$ $-1+O\left(\tau_{n}\right)$. Кроме того, $\nu_{n}=1+O\left(\tau_{n}\right)$. Поэтому

$$
\mathrm{E}\left(T_{n}\right) \underset{n \rightarrow \infty}{\sim} \frac{\sqrt{2} \sigma^{3} n^{3}}{\sqrt{\pi n} b_{n}^{3}} e^{n h_{n}\left(t_{n}\right)},
$$

что и доказывает утверждение следствия 1.

Авторы благодарны А.А. Могульскому и особенно анонимному рецензенту за ряд замечаний и советов, вследствие которых в работу была включена теорема 1.

\section{Список литературы}

1. В. Феллер, Введение в теорию вероятностей и ее приложения, т. 2, Мир, М., 1984, 752 с.; пер. с англ.: W. Feller, An introduction to probability theory and its applications, v. II, 2nd ed., John Wiley \& Sons, Inc., New York-London-Sydney, 1971, xxiv+669 pp.

2. Ф. Спицер, Принципъ случайного блуждания, Мир, М., 1969, 472 с.; пер. с англ.: F. Spitzer, Principles of random walk, The University Series in Higher Mathematics, D. Van Nostrand Co., Inc., Princeton, N.J.-Toronto-London, 1964, xi+406 pp.

3. А. В. Скороход, Н. П. Слободенюк, Предельные теоремы для случайных блужданий, Наукова думка, Киев, 1970, 303 с.

4. А. Н. Бородин, И. А. Ибрагимов, "Предельные теоремы для функционалов от случайных блужданий", Тр. МИАН СССР, 195, Наука, СПб., 1994, 3-285; англ. пер.: A. N. Borodin, I. A. Ibragimov, "Limit theorems for functionals of random walks", Proc. Steklov Inst. Math., 195 (1995), 1-259.

5. В.И. Лотов, “Асимптотические разложения распределения времени пребывания случайного блуждания на полуоси", Ветвящиеся прочессы, случайные блуждания и смежные вопросы, Сборник статей. Посвящается памяти члена-корреспондента РАН Бориса Александровича Севастьянова, Тр. МИАН, 282, МАИК, М., 2013, 154-164; англ. пер.: V. I. Lotov, "Asymptotic expansions for the distribution of the sojourn time of a random walk on a half-axis", Proc. Steklov Inst. Math., 282 (2013), 146-156.

6. А.А. Боровков, "Новые предельные теоремы в граничных задачах для сумм независимых слагаемых", Сиб. матем. журн., 3:5 (1962), 645-694; англ. пер.: A. A. Borovkov, "New limit theorems in boundary problems for sums of independent terms", Select. Transl. Math. Stat. Probab., 5 (1965), 315-372. 
7. В.И. Лотов, "Асимптотический анализ распределений в двуграничных задачах. II", ТВП, 24:4 (1979), 873-879; англ. пер.: V. I. Lotov, "Asymptotic analysis of distributions in problems with two boundaries. II", Theory Probab. Appl., 24:4 (1979), 869-876.

8. В.И. Лотов, "Предельные теоремы в одной граничной задаче для случайных блужданий”, Сиб. матем. журн., 40:5 (1999), 1095-1108; англ. пер.: V. I. Lotov, "Limit theorems in a boundary crossing problem for random walks", Siberian Math. J., 40:5 (1999), 925-937.

9. В.И. Лотов, "Факторизационные тождества для времени пребывания случайного блуждания в полосе", Сиб. матем. журн., 51:1 (2010), 146-155; англ. пер.: V. I. Lotov, "Factorization identities for the sojourn time of a random walk in a strip", Siberian Math. J., 51:1 (2010), 119-127.

10. В. И. Лотов, "О времени пребывания случайного блуждания в полосе", Сиб. матем. журн., 51:4 (2010), 785-804; англ. пер.: V. I. Lotov, "On the sojourn time of a random walk in a strip", Siberian Math. J., 51:4 (2010), 621-638.

11. А.А. Боровков, Теория вероятностей, 5-е изд., ЛИБРОКОМ, М., 2009, 656 с.; англ. пер.: А. А. Borovkov, Probability theory, Universitext, Springer, London, 2013, xxviii+733 pp.

12. А.А. Боровков, К.А. Боровков, Асимптотический анализ случайных блужданий, т. 1: Медленно убывающие распределения скачков, Физматлит, М., 2008, 652 с.; англ. пер.: А. A. Borovkov, K. A. Borovkov, Asymptotic analysis of random walks, Encyclopedia of Mathematics and its Applications, no. 118, Cambridge Univ. Press, Cambridge, 2008, xxx+625 pp.

13. Н. Г. де Брёйн, Асимптотические методы в анализе, ИЛ, М., 1961, 248 с.; пер. с англ.: N. G. de Bruijn, Asymptotic methods in analysis, Bibliotheca Mathematica, 4, North-Holland Publishing Co., Amsterdam; P. Noordhoff Ltd., Groningen; Interscience Publishers Inc., New York, 1958, xii+200 pp.

ВЛАДИМИР ИвАНОВИЧ ЛОТОв

Поступило в редакцию

(Vladimir I. Lotov)

15.10 .2013

Институт математики им. С. Л. Соболева СО РАН, 29.09.2014

г. Новосибирск;

Новосибирский государственный университет

E-mail: lotov@math.nsc.ru

Анtoh Cергеeвич Tapacehko

(Anton S. TARAsenko)

Институт математики им. С. Л. Соболева СО РАН, г. Новосибирск;

Новосибирский государственный университет

E-mail: dkanus@gmail.com 